Bryn Mawr College

Scholarship, Research, and Creative Work at Bryn Mawr

College

1976

\title{
Nuclear spin relaxation by intramolecular interactions in gases of homonuclear diatomic molecules
}

Myer Bloom

Peter A. Beckmann

Bryn Mawr College, pbeckman@brynmawr.edu

B C. Sanctuary

Let us know how access to this document benefits you.

Follow this and additional works at: https://repository.brynmawr.edu/physics_pubs

Part of the Physics Commons

\section{Custom Citation}

Bloom, Myer, Peter A. Beckmann, and B. C. Sanctuary. 1976. "Nuclear spin relaxation by intramolecular interactions in gases of homonuclear diatomic molecules." Canadian Journal of Physics 54.22: 2209-2212.

This paper is posted at Scholarship, Research, and Creative Work at Bryn Mawr College. https://repository.brynmawr.edu/physics_pubs/120

For more information, please contact repository@brynmawr.edu. 


\title{
Nuclear spin relaxation by intramolecular interactions in gases of homonuclear diatomic molecules
}

\author{
Myer Bloom, Peter Beckmann, and B.C. Sanctuary
}

Canadian Journal of Physics, 1976, 54.22: 2209-2212. https://doi.org/10.1139/p76-264

\begin{abstract}
The differential equations which describe the relaxation of macroscopic observables associated with nuclear spins in homonuclear diatomic molecules are derived using an expansion of the nuclear spin density matrix in terms of irreducible tensors. It is shown, using an intramolecular quadrupole mechanism, that the only difference between nuclear spin relaxation of the ortho- and para-species arises from the rotational states being restricted to odd and even values. This difference is vanishingly small at high temperatures so that the relaxation equations for nuclear magnetization become identical for both species. A previous paper predicting a difference even at high temperatures is shown to be in error and is corrected.
\end{abstract}

In an earlier paper by one of us (Sanctuary 1973), the nuclear spin relaxation time $T_{1}$ was derived for the ortho- and para-species of a homonuclear diatomic molecule. Using an intramolecular quadrupole interaction, it was found that the two species relax at different rates for $I>1$ even at temperatures high enough that averages of molecular quantities over the odd and even rotational states are approximately equal. The purpose of this paper is to show that this result is incorrect, that the relaxation, in general, is not exponential and that the relaxation equations for the two nuclear spin symmetry species are identical to each other at high temperatures. In fact, for intramolecular quadrupolar interactions we show that the relaxation equations for the magnetization of the ortho- and para-species are identical to each other and completely equivalent to those of two uncorrelated nuclear spins. Thus the only role played by the identical spins is via averages of molecular quantities over the rotational states of the two species.

The intramolecular quadrupolar Hamiltonian $\mathscr{H}_{\mathrm{Q}}$ for a diatomic molecule is given by (Abragam (1961), p. 233)

$$
\mathscr{H}_{\mathrm{Q}}=K \sum_{q=-2}^{+2} A_{2 q} Y_{2 q}^{*}(\theta, \phi)
$$

where

[2] $K=\frac{\sqrt{\pi}}{10} e q Q\left[\frac{(I+1)(2 I+1)(2 I+3)}{I(2 I-1)}\right]^{1 / 2}$

and

$$
A_{2 q}=\hat{\top}_{2 q}\left(I_{1}\right) E_{2}+E_{1} \hat{\top}_{2 q}\left(I_{2}\right)
$$

$Y_{2 q}(\theta, \varphi)$ is a spherical harmonic of the angles $\theta, \varphi$ associated with the vector joining the two nuclei and $I=I_{1}=I_{2}$ is the spin of the each nuccleus. The quadrupolar coupling constant is $e q Q$ and $E_{\mathrm{i}}$ is the identity operator for the spin degrees of freedom of nucleus $i$. Finally, $T_{2 q}\left(I_{\mathrm{i}}\right)$ is the $q$ th spherical component of the normalized irreducible tensor of rank 2 associated with the spin $I_{\mathrm{i}}$ of nucleus $i$. It is given explicitly by 


$$
\begin{aligned}
& \text { [4] } \hat{\mathrm{T}}_{2 q}\left(I_{i}\right)=\frac{\sqrt{30} \mathrm{~T}_{2 q}\left(I_{i}\right)}{[I(I+1)(2 I+3)(2 I+1)(2 I-1)]^{1 / 2}}
\end{aligned}
$$

where $\mathrm{T}_{2 q}\left(I_{i}\right.$ is the unnormalized spherical tensor components defined by, e.g., Rose (1957) and Tinkham (1964). Explicitly, $\mathrm{T}_{20}(I)=(6)^{-1 / 2}\left(3 I_{z}^{2}-I(I+1)\right)$. The normalization is chosen such that

$$
\operatorname{Tr}\left\{\hat{\top}_{L M^{\prime}} \hat{\top}_{L^{\prime} M^{\prime}}\right\}=\delta_{L L^{\prime}} \delta_{M M^{\prime}}
$$

In general, the $\hat{\top}_{L M}$ 's are found to be (Sanctuary

1976; Coope and Snider 1972)

$$
\begin{aligned}
& \text { [6] } \hat{\mathrm{T}}_{L M}\left(I_{i}\right)= \\
& {\left[\frac{(2 L+1) 2^{L}(2 L) !(2 I-L) !}{(L !)^{2}(2 I+L+1) !}\right]^{1 / 2} \mathrm{~T}_{L M}\left(I_{i}\right)}
\end{aligned}
$$

In the gas phase, nuclear spin relaxation is caused by molecular collisions which reorient the molecule thereby causing the rotational angular momentum $J$ to change direction, or, equivalently, producing transitions between rotational $m_{J}$ states. The effect of collision-induced molecular reorientation is to cause the angles $\theta, \varphi$ in [1] to be time dependent; thus inducing nuclear spin transitions via the spin-dependent interaction H $_{\mathrm{Q}}$ (Bloom 1972; Abragam 1961).

The relaxation equation for the spin density matrix $\sigma$ in a co-ordinate system rotating about the applied magnetic field at the nuclear spin Larmor frequency $\omega_{0}$ has been derived using time dependent perturbation theory to be (Abragam (1961), equation (42), p. 279

$$
\text { [7] } \frac{\mathrm{d} \tilde{\sigma}}{\mathrm{d} t}=-\frac{K^{2}}{2 \hbar^{2}} \sum_{q=-2}^{+2} J_{q}\left(q \omega_{0}\right)\left[A_{2 q}^{\dagger},\left[A_{2 q}, \tilde{\sigma}\right]\right]
$$

where $J_{q}(\omega)$ is the spectral density of the correlation function of $Y_{2 q}(\theta, \varphi)$.

It is convenient for our purposes to expand $\sigma$, the density matrix in the laboratory frame, in terms of the tensors $\mathrm{T}_{L M}\left(I_{i}\right)$ for each nucleus

$$
\text { [8] } \begin{aligned}
\sigma(t)= & \sum_{L, L^{\prime}=0}^{2 I} \sum_{M=-L}^{+L} \sum_{M^{\prime}==-L^{\prime}}^{+L^{\prime}} \\
& \times a_{M M^{\prime}}^{L L^{\prime}} \cdot(t) \hat{\top}_{L M}^{\prime}\left(I_{1}\right) \hat{T}_{L^{\prime} M^{\prime}}^{\dagger}\left(I_{2}\right)
\end{aligned}
$$

The products $\mathrm{T}_{L M}\left(I_{i}\right) \mathrm{T}_{L^{\prime} M^{\prime}}\left(I_{2}\right)$ form a complete set in the $\left(2 I_{i}+1\right)\left(2 I_{2}+1\right)$ dimensional nuclear spin space of the diatomic molecule. The representation of the density matrix in terms of products of irreducible tensors is commonly used in relaxation problems in atomic physics (Happer 1972) and in the kinetic theory of polyatomic gases (Ferziger and Kaper 1972). From the orthonormal properties of the irreducible spherical tensors, it may be seen that a macroscopic observable $\langle Q\rangle$ associated with a quantum mechanical operator $Q=\mathrm{T}_{i m}\left(I_{1}\right) \mathrm{T}_{l^{\prime} m} \cdot\left(I_{2}\right)$ has a value

$$
\begin{aligned}
\langle Q\rangle=\operatorname{Tr}(Q \sigma) & =a_{m m^{\prime}}^{l^{\prime}}(t) \\
& =\tilde{a}_{m m^{\prime}}^{l^{\prime}}(t) \mathrm{e}^{i\left(m+m^{\prime}\right) \omega_{0} t}
\end{aligned}
$$

We separate $\sigma$ into three sets of terms

$$
\sigma=\sigma_{\mathrm{o}}+\sigma_{\mathrm{s}}+\sigma_{\mathrm{c}}
$$


The first term is just the product of the two identity operators $\sigma_{0}=E_{1} E_{2}$, i.e. $L=L^{\prime}=0$ The second term is a 'single spin term' associated with the values of the indices $L=0, L^{\prime} \neq 0$ and $L \neq 0, L^{\prime}=0$. The coefficient $a \frac{L O}{M O}$ and $a \frac{O L^{\prime}}{O M}$, therefore, represent macroscopic quantities involving single spin quantities. In fact, the term $a \frac{L O}{M O}$ involves only observables associated with $I_{i}$ and $a \frac{O L^{\prime}}{O M}$, only those of $I_{2}$ so that the spin density matrix terms associated with them may be denoted by $\sigma_{\mathrm{s} 1}(t)$ and $\sigma_{\mathrm{s} 2}(t)$ respectively, with no ambiguity. The third term involves products of irreducible tensors in which both $L$ and $L^{\prime}$ are non-zero. Their coefficients $a \frac{L L^{\prime}}{M M}$ are associated with macroscopic observables which depend on correlations between the two spins.

Since all $\mathrm{T}_{L M}\left(I_{i}\right)$ commute with all $\mathrm{T}_{L M}\left(I_{\mathrm{j}}\right)$ for $i \neq j$, and since $E_{i}$ commutes with all $\mathrm{T}_{L M}$ $\left(I_{\mathrm{j}}\right)$ for all $i, j$, it is clear that

$$
\left[\sigma_{31}, E_{1} \hat{\top}_{L M}\left(I_{2}\right)\right]=\left[\sigma_{s 2}, \hat{\top}_{L M}\left(I_{1}\right) E_{2}\right]=0
$$

for any $L M$. In addition, $\left[\sigma_{s 1}, \hat{T}_{L M}\left(I_{1}\right) E_{2}\right]$ involves only operators of the type $\hat{T}_{L^{\prime} M}\left(I_{1}\right) E_{2}$.

Substituting [3] and [8] into [7] and using [9],

[10], and [11], it follows that

$$
\text { [12] } \frac{\mathrm{d}}{\mathrm{d} t} \tilde{a}_{M O}^{L O}=-\sum_{L^{\prime \prime M} M^{\prime \prime}} b_{L L^{\prime \prime} M M^{\prime \prime}} \tilde{a}_{M^{\prime \prime}}^{L^{\prime \prime O} O}(t)
$$

where the coefficients

$$
b_{L L^{\prime \prime} M M^{\prime \prime}}=\frac{K^{2}}{\partial \hbar^{2}} \sum^{+2} J_{q}\left(q \omega_{0}\right) \operatorname{Tr}\left\{\left[\hat{\mathrm{T}}_{L M}(I), \hat{\mathrm{T}}_{2 q}^{\dagger}(I)\right]\left[\hat{\mathrm{T}}_{2 q}(I), \hat{\mathrm{T}}_{L^{\prime \prime} M^{\prime \prime}}(I)\right]\right\}
$$

are identical to those obtained in the corresponding differential equation fora $\tilde{a} \frac{O L}{O M}(t)$. Thus the relaxation equations obtained for $\sigma_{\mathrm{s} 1}$ and $\sigma_{\mathrm{s} 2}$ are identical with each other and to their sum $\sigma_{\mathrm{s}}=$ $\sigma_{\mathrm{s} 1}+\sigma_{\mathrm{s} 2}$. It follows that the relaxation equations for nuclear magnetization for the two-spin molecule are formally equivalent for quadrupolar relaxation to those of a single spin. The only difference between the relaxation rates of macroscopic observables represented by operators of the form $Q=\mathrm{T}_{i m}\left(I_{1}\right) E_{2}+E_{1} \mathrm{~T}_{i m}\left(I_{2}\right)$ such as magnetization $(I=1)$, for the ortho- and para-species arises from averages over the different rotational states of the two species of the molecular parameters arising from the terms in $J_{q}\left(q \omega_{0}\right)$ in [7]. These differences become vanishingly small at high temperatures.

The commutators in [13] can be explicitly calculated (see, e.g, Sanctuary (1976), equation (46); Rose (1962); Ambler et al. (1962)) and the resulting expression written $\mathrm{m}$ terms of 3-j and 6-j coefficients (Edmond 1961),

$$
\begin{aligned}
b_{L L^{\prime \prime} M M^{\prime \prime}}= & \delta_{M M^{\prime \prime}} \frac{\pi}{10}\left(\frac{e q Q}{\hbar}\right)^{2}\left[\frac{(I+1)(2 I+1)(2 I+3)}{I(2 I-1)}\right] \sum_{2=0}^{2 I} \phi(\lambda 2 L) \phi\left(\lambda 2 L^{\prime \prime}\right)(2 \lambda+1) \\
& \times\left\{\begin{array}{lll}
L & 2 & \lambda \\
I & I & I
\end{array}\right\}\left\{\begin{array}{ccc}
L^{\prime \prime} & 2 & \lambda \\
I & I & I
\end{array}\right\} \sum_{q=-2}^{2} J_{q}\left(q \omega_{0}\right)\left[(2 L+1)\left(2 L^{\prime \prime}+1\right)\right]^{1 / 2} \\
& \times\left(\begin{array}{cccc}
L & 2 & \lambda \\
-M & q & M-q
\end{array}\right)\left(\begin{array}{ccc}
L^{\prime \prime} & 2 & \lambda \\
-M & q & M-q
\end{array}\right)
\end{aligned}
$$

The quantity $\varphi\left(l_{1}, l_{2}, l_{3}\right)$ is equal to unity if the sum $l_{1}+l_{2}+l_{3}$ is odd and zero otherwise Hence $L$ and $L$ ' are either both even or both odd. Moreover the $b_{L L}{ }^{\prime} M{ }^{\prime}$,'s obey the symmetry relation

$$
b_{L L^{\prime \prime M M}}=b_{L^{\prime \prime} L M M}
$$


For $I=1$, the relaxation of the magnetization $(L=1)$ is exponential since only $L "=1$ contributes to [14]. In this case $1 / T_{1}$, is given by $b_{1100}$ with $I=1, \lambda=2$. It is easily shown that this form agrees with Abragam (1961, equation (138), p. 314). For $I=3 / 2$ and $2, L=1$ and 3 contribute to the nuclear spin relaxation giving rise to a sum of two exponentials. Spins of $5 / 2$ and greater involve coupling to multiples of rank $L^{\prime \prime}=5$ and higher and are generally nonexponential. On the other hand, as shown by Pyper (1971), in the extreme narrowing limit, the $q$ dependence of $J_{q}\left(q \omega_{0}\right)$ can be neglected resulting in exponential decay for spins of any magnitude. This is seen from the orthogonality of the $3-j$ 's in [14] giving $L=L "$. In this case $1 / T_{1}$ is given by $b_{1100}$ with $\lambda=2$ for any $I$ and agrees with the expression given by Abragam ((1961), equation (137), p. $314)$.

In a previous paper (Sanctuary 1973), the problem of nuclear spin relaxation $m$ orthoand paraspecies was treated by evaluating the relaxation tensor for the $s \sim$ th species $(1 / T)_{s}$ (equation (19) of Sanctuary (1973)). This procedure assumes that the Bloch equations are obeyed from the outset and focuses attention on the different spin weightings due to ortho-para restrictions. It was found, in contradiction to the calculation here, that the ortho- and para-species have different relaxation times even at high temperatures. This conclusion was obtained due to an error in normalization in [22] of Sanctuary (1973). The correct result is

$$
\text { [16] }\left\langle P_{s} I \cdot P_{s} I\right\rangle=2 \sum_{I, I^{\prime}}^{\prime}\left(1+(-1)^{I+I^{\prime}}\right)(2 I+1)\left(2 I^{\prime}+1\right)\left\{\begin{array}{lll}
I & I & I^{\prime} \\
I_{1} & I_{1} & I_{1}
\end{array}\right\}^{2}\left\langle I_{1}\left\|I_{1}\right\| I_{1}\right\rangle^{2}
$$

where $/$, / ' are the total spin magnitudes of the resultant $I=I_{1}+I_{2}$ with values that range from 0 to $2 / I_{1}$. The prime on the summation restricts these to either odd or even integers as symmetry requires. Carrying the expression [16] through the calculation subsequent to [22] in Sanctuary (1973) gives the result $1 / T_{1}-b_{1100 i n}$ agreement with the expression developed in this paper.

ABRAGAM, A. 1961. The principles of nuclear magnetism (Oxford University Press, London). AMBLER, E., BISENSTEIN, J. C, and SCHOOLEY, J. F. 1962. J. Math. Phys. 3, 118,760.

BLOOM, M. 1972. MTP international review of science, physical science series one. Vol. 4, ed. by C. A. McDowell (Butterworth, London), p. 1.

COOPE, J. A. R. and SNIDER, R. F 1972. J. Chem. Phys. 56,2049.

EDMOND, A. R. 1960. Angular momentum in quantum mechanics (Princeton UP, Princeton, $\mathrm{NJ})$.

FERZIGER, J. H. and KAPER, H. G. 1972. Mathematical theory of transport processes in gases (North Holland Publishing Company, Amsterdam).

HAPPER, W. 1972. Rev. Mod. Phys. 44, 169.

PYPER,N.C. 1971. Mol. Phys. 21,1.

ROSE, M. E. 1957. Elementary theory of angular momentum (John Wiley and Sons, New York, NY).

ROSE, M. E. 1962. J. Math. Phys. 3,409.

SANCTUARY, B . C. 1973. Can. J. Phys. 51,2488. 1976. J. Chem. Phys. 64,4352.

TINKHAM, M. 1964. Group theory and quantum mechanics (McGraw-Hill, New York, NY). 